\title{
O nexo entre religiosidade/espiritualidade e o comportamento suicida em jovens*
}

\author{
Maria Betânia Tinti de Andrade ${ }^{1}$ \\ (D) https://orcid.org/0000-0003-0329-1299 \\ Andriana Olimpia Barbosa Felipe ${ }^{1}$ \\ (D) https://orcid.org/0000-0002-4491-5750 \\ Kelly Graziani Giacchero Vedana ${ }^{2}$ \\ (1D) https://orcid.org/0000-0001-7363-2429 \\ Fabio Scorsolini-Comin ${ }^{2}$ \\ (D) https://orcid.org/0000-0001-6281-3371
}

Objetivo: avaliar as evidências científicas referentes à relação entre religiosidade/espiritualidade (R/E) e o comportamento suicida. Método: estudo de revisão integrativa da literatura, nas bases de dados: LILACS, PubMed e CINAHL, de janeiro de 2011 a fevereiro de 2020. Foram identificados 1044 artigos, dos quais sete foram incluídos no estudo. Resultados: Predominaram estudos quantitativos (71,4\%), realizados com universitários $(57,14 \%)$, que investigaram a religião $(71,43 \%)$ e diferentes dimensões do comportamento suicida $(85,7 \%)$. A maioria dos estudos retratara benefícios da religião e da espiritualidade, mesmo que indiretamente na vida dos jovens e apontaram para efeitos protetivos da R/E em relação ao comportamento suicida. Conclusão: A R/E pode ser um fator importante para adolescentes e adultos jovens que merece ser explorada em investigações e ações que fortaleçam os fatores protetores contra o comportamento suicida e permitam o aumento da literacia em saúde mental entre lideranças religiosas.

Descritores: Espiritualidade; Religião; Adolescente; Jovem Adulto; Tentativa de Suicídio; Ideação Suicida.

\section{Como citar este artigo}

Andrade MBT, Felipe AOB, Vedana KGG, Scorsolini-Comin F. The nexus between religiosity/spirituality and suicidal behavior in young people. SMAD, Rev Eletrônica Saúde Mental Álcool Drog. 2020;16(4):109-121. doi: https://dx.doi.org/10.11606/issn.1806-6976.smad.2020.169257 


\section{The nexus between religiosity/spirituality and suicidal behavior in young people}

Objective: to evaluate the scientific evidence regarding the relationship between religiosity/ spirituality (R/S) and suicidal behavior. Method: an integrative literature review study, conducted in the following databases: LILACS, PubMed and CINAHL, from January 2011 to February 2020. A total of 1,044 articles were identified, of which seven were included in the study. Results: quantitative studies (71.4\%), carried out with university students (57.14\%), which investigated religion (71.43\%) and different dimensions of suicidal behavior (85.7\%) predominated. Most of the studies portrayed the benefits of religion and spirituality, even if indirectly in the lives of young people and pointed to the protective effects of R/S in relation to suicidal behavior. Conclusion: R/S can be an important factor for adolescents and young adults that deserve to be explored in investigations and actions that strengthen the protective factors against suicidal behavior and allow for an increase in mental health literacy among religious leaderships.

Descriptors: Spirituality; Religion; Adolescent; Young Adult; Attempted Suicide; Suicidal Ideation.

\section{El nexo entre religiosidad/espiritualidad y comportamiento suicida en los jóvenes}

Objetivo: evaluar la evidencia científica sobre la relación entre religiosidad/espiritualidad (R/E) y conducta suicida. Método: revisión integradora de la literatura, en las bases de datos: LILACS, PubMed y CINAHL, de enero de 2011 a febrero de 2020. Se identificaron 1044 artículos, de los cuales, siete fueron incluidos en el estudio. Resultados: predominaron los estudios cuantitativos $(71,4 \%)$, realizados con estudiantes universitarios $(57,14 \%)$, que investigaron religión $(71,43 \%)$ y diferentes dimensiones de la conducta suicida $(85,7 \%)$. La mayoría de los estudios han retratado los beneficios de la religión y la espiritualidad, incluso indirectamente en la vida de los jóvenes, y han señalado los efectos protectores de la R/E en relación con la conducta suicida. Conclusión: la R/E puede ser un factor importante para los adolescentes y adultos jóvenes que merece ser explorado en investigaciones y acciones que fortalezcan los factores protectores contra la conducta suicida y permitan un aumento de los conocimientos en salud mental entre los líderes religiosos.

Descriptores: Espiritualidad; Religión; Adolescente; Joven Adulto; Intento de Suicidio; Ideación Suicida. 


\section{Introdução}

De acordo com dados da Organização Mundial da Saúde (OMS), estima-se que a cada 40 segundos uma pessoa morre por suicídio em algum lugar do mundo, o que corresponde a mais de 800.000 óbitos por ano. O suicídio é um problema particularmente preocupante entre adolescentes e jovens adultos, pois é a segunda principal causa de morte na idade de 15 a 29 anos. Considerando que o suicídio é multifatorial, múltiplas ações podem ser realizadas para preveni-lo(1). A atenção a essa faixa etária dos 15 a 29 anos, que compreende a transição da adolescência para o início da vida adulta, vem se mostrando, portanto, um foco importante para as investigações, intervenções e políticas de prevenção ao suicídio.

O comportamento suicida é um fenômeno complexo que está associado a um conjunto de ideias, intenções e ações relacionados ao desejo de provocar a própria morte $^{(2)}$. Na juventude, diferentes fatores podem contribuir para uma maior exposição dessa população a esse fenômeno, como variáveis relacionadas ao trabalho, à vida familiar, às vivências grupais, a quadros psicopatológicos pré-existentes, ao maior consumo de substâncias, às perspectivas de futuro e aos próprios conflitos oportunizados nessa fase do desenvolvimento, que marca a transição entre a adolescência e os desafios da vida adulta, representando um período de maior mobilização emocional e também de maior fragilidade diante dos diversos desafios. Além disso, o fenômeno do suicídio nessa faixa etária também deve ser compreendido a partir das suas repercussões nas redes de apoio social, compondo um fenômeno que atinge, de modo especial, a família(3-4).

Em que pesem os expressivos dados epidemiológicos, que asseveram o suicídio como uma das três principais causas de morte entre jovens, algumas estratégias de prevenção e de posvenção ao suicídio têm sido cada vez mais discutidas na literatura científica ${ }^{(1)}$, a exemplo da religiosidade/espiritualidade (R/E). Estudos que comparam a R/E de jovens adultos e de idosos destacam que os efeitos dessa variável na saúde podem ser mais expressivos entre a população mais idosa(5). Isso nos leva à necessidade de compreender melhor como os jovens se posicionam em relação às vivências da R/E e o papel da mesma nos desfechos em saúde dessa população.

Estudos apontam evidências acerca das relações positivas entre R/E e saúde mental(6-8) e também sua influência na saúde física das pessoas com diversas alterações no processo saúde-doença(9-10). Contudo, as relações entre a R/E e o comportamento suicida ainda são incongruentes(11), sobretudo quando analisamos adolescentes e jovens adultos. Conhecer como esse público vivencia a $R / E$ e se esse fator pode ser protetivo no contexto da saúde mental, sobretudo na prevenção ao suicídio, apresenta-se como uma lacuna importante de ser investigada.

Neste contexto, é relevante compreender que a R/E é uma nomenclatura especialmente utilizada no contexto da saúde e que abrange, de forma combinada, as definições de religiosidade, espiritualidade e também de religião. Em que pesem as diferenças semânticas e epistemológicas entre esses termos, a adoção do termo combinado, $\mathrm{R} / \mathrm{E}$, tem sido útil nos estudos que enfocam os efeitos dessa dimensão no cuidado em saúde, envolvendo questões relacionadas ao sentido da vida e ao sagrado, podendo ou não levar às práticas religiosas e institucionais(12-13). A dimensão da R/E é parte integrante do indivíduo, levando-nos a reflexões sobre como a mesma pode repercutir no contexto da saúde e de que maneira pode influenciar como fator de proteção para o comportamento suicida. A partir dessas considerações, o presente estudo teve como objetivo avaliar as evidências científicas referentes à relação entre R/E e o comportamento suicida em adolescentes e jovens adultos.

\section{Método}

Trata-se de um estudo de revisão integrativa da literatura, que tem como finalidade identificar e analisar as evidências científicas de forma sistemática e ordenada, para conseguir o aperfeiçoamento de conhecimento do tema pesquisado e a necessidade de novas pesquisas ${ }^{(14)}$. Para este estudo, optou-se pelas seguintes etapas: Identificação de um problema, formulação de uma questão norteadora, busca das evidências científicas, avaliação das evidências disponíveis ${ }^{(15)}$.

Quanto à elaboração da questão norteadora, utilizou-se a estratégia PICO, composta pelo acrônimo ligado aos seguintes itens (Patient: adolescente e jovem adulto de 15 a 24 anos; Intervention: R/E Comparison: pessoas sem R/E (nos estudos nos quais houve comparação); e Outcomes: comportamento suicida. Em relação à idade da população considerada para a presente revisão, a delimitação do período compreendido entre 15 e 24 anos teve por objetivo abarcar indivíduos adolescentes ou jovem adultos, que estão em uma faixa etária na qual o suicídio desponta como a segunda causa de mortalidade ${ }^{(1)}$, requerendo, portanto, ações preventivas e reconhecimento de fatores de risco e de proteção. Destaca-se que, segundo a OMS, o adolescente é o indivíduo que possui entre 10 e 19 anos $^{(16)}$, enquanto em classificações nacionais, o jovem adulto é aquele que possui entre 20 e $24 \operatorname{anos}^{(17)}$.

A estratégia PICO foi desenvolvida de modo alinhado à prática baseada em evidências para a localização de estudos primários relevantes nas bases/ bibliotecas de dados ${ }^{(15)}$. Na presente revisão, a questão 
norteadora foi: Quais as evidências científicas referentes à associação entre R/E e comportamento suicida entre adolescentes e jovens adultos?

A coleta de dados foi realizada entre os meses de fevereiro e março de 2020. Para realização da busca optou-se pelas bases/bibliotecas LILACS (Literatura Latino-americana e do Caribe em Ciências da Saúde), PubMed (National Library of Medicine and National Institute of Health - USA), CINAHL (The Cumulative Index to Nursing and Allied Health Literature). Tais bases/ bibliotecas foram selecionadas devido à sua abrangência e relevância para a identificação de evidências, como reportado em outras revisões de literatura disponíveis ${ }^{(18)}$.

Utilizou-se os termos indexados nos descritores de Ciências da Saúde (DECs) e Medical Subject Headings (MeSH). Foi utilizada a seguinte combinação dos descritores indexados no DECs e MeSH Terms: ideação suicida (suicidal ideation), suicídio (suicide), tentativa de suicídio (suicide attempted), espiritualidade (spirituality), religião (religion), adolescente (adolescent) e jovem adulto (young adult). Além da utilização da palavra chave jovem (young). Foi empregado o operador booleano AND, perfazendo dezoito combinações de buscas diferentes. Como estratégia de busca, incluiu-se a pesquisa dos descritores e da palavra-chave ao longo do texto. Esses descritores e suas combinações foram definidos a partir da pergunta norteadora.

Foram utilizados como critérios de inclusão: artigos em português, espanhol e inglês, publicados no período de janeiro de 2011 a fevereiro de 2020, que retratassem a relação entre a R/E e o comportamento suicida, tendo como participantes adolescentes e jovens adultos com idade entre 15 a 24 anos. O período de abrangência da revisão, abarcando o recorte dos últimos nove anos, visou a possibilitar o acesso à produção mais recente acerca do tema.

Essa idade entre 15 a 24 anos foi selecionada por englobar adolescentes e jovens adultos que estão na faixa etária da população com maior vulnerabilidade para o comportamento suicida(1).

Quantos aos critérios de exclusão, foram retirados artigos de revisão de literatura, editoriais, resumos, artigos repetidos na base de dados, cujos textos não estavam disponíveis na íntegra, que não respondessem à questão norteadora, artigos que abordavam outras comorbidades e cujos participantes estivessem abaixo dos 15 anos e acima de 25 anos de idade, que informassem apenas a média de idade ou que não mencionassem a idade dos participantes da pesquisa.

As buscas e a aplicação dos critérios de inclusão e de exclusão foram realizadas por dois juízes independentes, ambos com experiência nas temáticas da R/E e do suicídio, bem como com familiaridade com o método de revisão adotado. As possíveis disparidades nessas avaliações foram apreciadas por um terceiro juiz. Os artigos recuperados a partir desse processo de filtragem e seleção compuseram o corpus analítico.

Para a categorização dos artigos foram extraídas as seguintes informações dos artigos que compuseram o corpus: autores, ano de publicação, título, periódico, idioma, objetivo, características metodológicas, instrumentos e questionários utilizados.

\section{Resultados}

Na Figura 1, observa-se o processo de seleção e dos critérios de exclusão e inclusão dos estudos. Primeiramente, foram identificados 1044 artigos nas bases de dados/bibliotecas selecionadas. Após a leitura e análise dos títulos identificando o tema principal como: comportamento suicida (ideação e tentativa de suicídio) e espiritualidade, religião ou religiosidade, foram préselecionados 433 artigos. Com a leitura dos títulos e resumos, foram excluídos 314 artigos e 112 artigos não atendiam aos critérios de inclusão, totalizando uma amostra final de sete artigos.

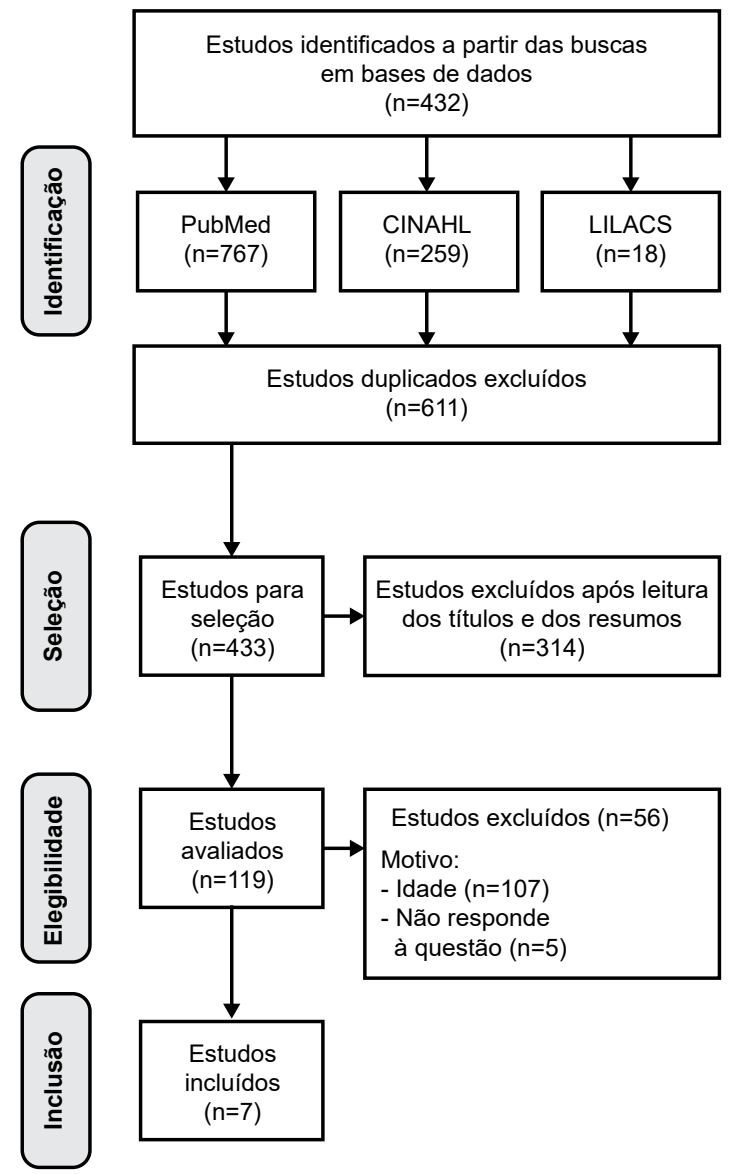

Figura 1 - Fluxograma do processo de seleção dos estudos para a revisão sistemática da literatura

As causas de exclusão foram: idade dos participantes $(n=107 ; 95,5 \%)$ e artigos que não abordavam a temática estudada $(n=5 ; 4,5 \%)$. 
O corpus do estudo foi composto por sete artigos que atenderam aos critérios de inclusão. A síntese de informação dos artigos recuperados está apresentada na Figura 2.

Constatou-se que $(73,47 \%)$ das publicações são da PubMed e $(24,81 \%)$ CINAHL. Quanto o ano de publicação, verificou-se que sete estudos selecionados foram publicados entre 2011 a 2017. Houve predomínio em 2011 com duas $(28,5 \%)$ publicações e em 2012, 2014, 2015, 2016 e 2017, houve apenas uma (14,3\%). Em relação ao idioma de divulgação, sete (100\%) foram publicados em inglês e em revistas internacionais, sendo que três $(42,86 \%)$ artigos foram publicados em um mesmo periódico, o Journal of Religion and Health(19, 20,22).

Na Figura 3, encontra-se um panorama geral das sete publicações selecionadas, destacando-se o tipo de abordagem metodológica, a amostra, a idade dos participantes, o cenário e o objetivo geral.

Quanto ao delineamento das pesquisas, identificou-se que cinco estudos $(71,4 \%)$ foram de abordagem metodológica quantitativa e dois $(28,6 \%)$ de abordagem qualitativa. Detectou-se que os estudos de abordagem quantitativa tiveram uma amostra que variou de 111 a 1168 participantes, sendo que os estudos foram conduzidos em diferentes cenários de investigação. Neste contexto, ressalta-se que nenhum dos estudos apresentou o cálculo do tamanho amostral. Nos dois $(28,6)$ artigos qualitativos, a amostra variou de 18 a 20 participantes ${ }^{(19,24)}$.

Em quatro estudos $(57,14 \%)$ o cenário de investigação se deu nas universidades, dois $(28,57 \%)$ se desenvolveram em escolas com jovens do ensino médio(21,25), e o outro com grupo religioso do Islan ${ }^{(20)}$. A idade dos participantes variou de 15 a 24 anos. Identificou-se também que seis $(85,7 \%)$ dos estudos abordaram jovens de ambos os sexos, e um (14,3\%) estudo qualitativo se desenvolveu apenas com as mulheres jovens ${ }^{(19)}$.
Com relação ao local do estudo, detectou-se que quatro $(57,1 \%)$ foram desenvolvidos na Ásia (Paquistão, China, Malásia, Índia), dois (26,8\%) no Oriente Médio (Israel) e um (14,3\%) na África do Sul (Durban) e na América do Norte (Canadá).

Ao analisar os objetivos dos estudos, é prudente mencionar que seis $(85,7 \%)$ pesquisas tinham como objetivo avaliar diretamente o construto religião/ espiritualidade. Ainda em relação aos objetivos, no quesito comportamento suicida, constatou-se que seis pesquisas investigaram dimensões diferenciadas do comportamento suicida, avaliaram as atitudes, a ideação, tendências suicidas e pensamentos. Vale ressaltar também que um $(14,3 \%)$ dos artigos avaliou indiretamente o risco suicida por meio das situações estressantes do dia-dia(19).

No que diz respeito aos instrumentos e questionamentos utilizados para avaliar a R/E e o comportamento suicida, a Figura 4 sumariza esses elementos na presente amostra.

Ao analisar os instrumentos utilizados para a temática em tela, evidenciou-se a aplicação de instrumentos e estratégias diversificadas. Constatou-se que cinco $(71,43 \%)$ estudos quantitativos analisaram a religião, e não a espiritualidade. E que a avaliação da religiosidade em dois $(28,57 \%)$ dos artigos foi realizada apenas com respostas dicotômicas, sendo que um avaliou apenas a crença religiosa(23), e o outro além da crença religiosa avaliou os rituais religiosos ${ }^{(21)}$, e um artigo identificou a importância da religião e a participação no serviço religioso(25).

Nas pesquisas qualitativas, um dos estudos investigou a espiritualidade com perguntas semiestruturada, as quais não foram explicitadas ${ }^{(19)}$, e no outro a avaliação da religiosidade ocorreu através da pergunta "o que significava ser religioso", e as demais perguntas da religiosidade estavam envolvidas com a vida, estratégias de enfrentamento e o suicídio(24).

\begin{tabular}{|c|c|c|c|c|}
\hline Bases & Autores & Título & Ano & Periódico \\
\hline PubMed & Francis W, Bance LO(19) & $\begin{array}{l}\text { Protective Role of Spirituality from the Perspective of } \\
\text { Indian College Students with Suicidal Ideation: "I am here } \\
\text { Because God Exists". }\end{array}$ & 2017 & J Relig Health \\
\hline CINAHL & Kazi TB, Naidoo $\mathrm{S}^{(20)}$ & $\begin{array}{l}\text { Does Religiosity Mediate Suicidal Tendencies? A South } \\
\text { African Study of Muslim Tertiary Students. }\end{array}$ & 2016 & J Relig Health \\
\hline CINAHL & Muallem HK, Israelashvilli M(21) & $\begin{array}{l}\text { Religiosity as a buffer against suicidal ideation: a } \\
\text { comparison between Christian and Muslim-Arab } \\
\text { adolescents }\end{array}$ & 2015 & $\begin{array}{l}\text { Mental Health, Religion \& } \\
\text { Culture }\end{array}$ \\
\hline PubMed & $\begin{array}{l}\text { Foo XY, Alwi MNM, Ismail SIF, } \\
\text { Ibrahim N, Osman ZJ(22) }\end{array}$ & $\begin{array}{l}\text { Religious Commitment, Attitudes Toward Suicide, and } \\
\text { Suicidal Behaviors Among College Students of Different } \\
\text { Ethnic and Religious Groups in Malaysia. }\end{array}$ & 2014 & J Relig Health \\
\hline CINAHL & $\begin{array}{l}\text { Zhao J, Yang X, Xiao R, Zhang } \\
\text { X, Aguilera D, Zhao J(23) }\end{array}$ & $\begin{array}{l}\text { Belief system, meaningfulness, and psychopathology } \\
\text { associated with suicidality among Chinese college } \\
\text { students: a cross-sectional survey. }\end{array}$ & 2012 & BMC Public Health \\
\hline CINAHL & $\begin{array}{l}\text { Band M, Dein S, Loewenthal } \\
\mathrm{K} \mathrm{M}^{(24)}\end{array}$ & $\begin{array}{l}\text { Religiosity, coping, and suicidality within the religious } \\
\text { Zionist community of Israela thematic qualitative analysis. }\end{array}$ & 2011 & $\begin{array}{l}\text { Mental Health, Religion \& } \\
\text { Culture }\end{array}$ \\
\hline CINAHL & $\begin{array}{l}\text { Rasic D, Kisely S, Langille } \\
\text { DB }^{(25)}\end{array}$ & $\begin{array}{l}\text { Protective associations of importance of religion and } \\
\text { frequency of servisse attendance with depression risk, } \\
\text { suicidal behaviours and substance use in adolescents in } \\
\text { Nova Scotia, Canada. }\end{array}$ & 2011 & $\begin{array}{l}\text { Journal of Affective } \\
\text { Disorders }\end{array}$ \\
\hline
\end{tabular}

Figura 2 - Síntese dos artigos publicados nas bases de dados PubMed e CINAHL, no período de 2011 a 2020 


\begin{tabular}{|c|c|c|}
\hline Abordagem metodológica & Amostra, idade e cenário & Objetivo \\
\hline $\begin{array}{l}\text { Qualitativo } \\
\text { Francis W, Bance LO(19) }\end{array}$ & $\begin{array}{l}20 \text { universitárias na Índia de uma única } \\
\text { universidade, do sexo feminino, com } \\
\text { idade entre } 19 \text { a } 22 \text { anos. }\end{array}$ & $\begin{array}{l}\text { Explorar as percepções das estudantes universitárias em relação } \\
\text { às crenças "espiritualidade" como um fator protetor que contribui } \\
\text { para a adaptação positiva durante as situações estressantes. }\end{array}$ \\
\hline $\begin{array}{l}\text { Quantitativo } \\
\text { Kazi TB, Naidoo S(20) }\end{array}$ & $\begin{array}{l}111 \text { universitários adeptos ao Islã de } \\
\text { várias instituições, na África do Sul, de } \\
\text { ambos os sexos com idade entre } 18 \text { a } \\
21 \text { anos. }\end{array}$ & $\begin{array}{l}\text { Investigar a relação entre religiosidade e a tendência suicida em } \\
\text { uma amostra de estudantes muçulmanos. }\end{array}$ \\
\hline $\begin{array}{l}\text { Quantitativo } \\
\text { Muallem HK, Israelashvilli M(21) }\end{array}$ & $\begin{array}{l}219 \text { adolescentes de escolas secundárias } \\
\text { de Israel, de ambos os sexos, com idade } \\
\text { entre } 15 \text { a } 18 \text { anos. }\end{array}$ & $\begin{array}{l}\text { Explorar a associação entre crenças religiosas e pensamentos } \\
\text { suicidas entre muçulmanos e adolescentes cristãos da minoria } \\
\text { árabe do Estado de Israel. }\end{array}$ \\
\hline $\begin{array}{l}\text { Quantitativo } \\
\text { Foo XY, Alwi MNM, Ismail SIF, } \\
\text { Ibrahim N, Osman ZJ(22) }\end{array}$ & $\begin{array}{l}139 \text { universitários de duas faculdades da } \\
\text { Malásia, de ambos os sexos, com idade } \\
\text { entre } 18 \text { a } 24 \text { anos. }\end{array}$ & $\begin{array}{l}\text { Examinar o impacto do compromisso religioso e atitudes em } \\
\text { relação ao suicídio e ao comportamento suicida. }\end{array}$ \\
\hline $\begin{array}{l}\text { Quantitativo } \\
\text { Zhao J, Yang X, Xiao R, Zhang } \\
\text { X, Aguilera D, Zhao J(23) }\end{array}$ & $\begin{array}{l}1168 \text { estudantes universitários na China } \\
\text { de dez instituições de ensino, de ambos } \\
\text { os sexos, com idade entre } 16 \text { a } 24 \text { anos. }\end{array}$ & $\begin{array}{l}\text { Investigar o efeito do sistema de crenças, incluindo crenças } \\
\text { políticas e religiosas, e sua interação com o comportamento } \\
\text { suicida. }\end{array}$ \\
\hline $\begin{array}{l}\text { Qualitativo Band M, Dein S, } \\
\text { Loewenthal K M M(24) }\end{array}$ & $\begin{array}{l}18 \text { participantes da comunidade sionista } \\
\text { de Israel, sendo } 16 \text { universitários e dois } \\
\text { não, de ambos os sexos, com idade entre } \\
18 \text { a } 24 \text { anos. }\end{array}$ & $\begin{array}{l}\text { Mapear os principais aspectos da relação entre comportamento } \\
\text { suicida, enfrentamento e religiosidade. }\end{array}$ \\
\hline $\begin{array}{l}\text { Quantitativo } \\
\text { Rasic D, Kisely S, Langille } \\
\mathrm{DB}^{(25)}\end{array}$ & $\begin{array}{l}1615 \text { estudantes de três escolas do } \\
\text { ensino médio do Canadá, de ambos os } \\
\text { sexos, com idade entre } 15 \text { a } 19 \text { anos. }\end{array}$ & $\begin{array}{l}\text { Examinar a relação entre duas dimensões da religião (importância } \\
\text { e a frequência de atendimento religioso) com o risco de } \\
\text { Depressão, o comportamento suicida e os transtornos do uso de } \\
\text { substâncias. }\end{array}$ \\
\hline
\end{tabular}

Figura 3 - Características dos artigos quanto às variáveis tipo de abordagem metodológica, amostra, idade dos participantes, gênero, cenário e objetivo

\begin{tabular}{|c|c|c|}
\hline & $\begin{array}{l}\text { Religião/Espiritualidade } \\
\text { Autores validação ou construção }\end{array}$ & $\begin{array}{l}\text { Comportamento suicida } \\
\text { Autores validação ou construção }\end{array}$ \\
\hline Francis W, Bance LO ${ }^{(19)}$ & $\begin{array}{l}\text { Realizada através de grupo focal com } \\
\text { perguntas semiestruturadas para reunir } \\
\text { informações sobre a espiritualidade e os } \\
\text { fatores que contribuem para a adaptação } \\
\text { positiva. }\end{array}$ & $\begin{array}{l}\text { Quais são os estressores que você experimenta que o } \\
\text { levam a ter pensamentos de acabar com sua vida? } \\
\text { Quais são os fatores que ajudam você a ser forte } \\
\text { enfrentando as dificuldades/estressores que você encontra } \\
\text { em sua vida? } \\
\text { Como você vê a crença no supremo Criador, uma fonte de } \\
\text { força? }\end{array}$ \\
\hline Kazi TB, Naidoo, $\mathrm{S}^{(20)}$ & ROT - Teste de orientação religiosa ${ }^{(26)}$. & $\begin{array}{l}\text { MAST - Escala de Tendências ao suicídio de múltiplas } \\
\text { atitudes }^{(27)}\end{array}$ \\
\hline Muallem HK, Israelashvilli M(21) & Questionário de Religiosidade ${ }^{(28)}$. & Questionário de Ideação suicida ${ }^{(29)}$ \\
\hline $\begin{array}{l}\text { Foo XY, Alwi MNM, Ismail SIF, } \\
\text { Ibrahim N, Osman ZJ(22) }\end{array}$ & $\begin{array}{l}\mathrm{RCl}-10 \text { - Inventário de compromisso } \\
\text { religioso }{ }^{(30)} \text {. }\end{array}$ & ATTS - Atitudes em relação a Escala de Suicídio(31) \\
\hline $\begin{array}{l}\text { Zhao J, Yang X, Xiao R, Zhang } \\
\text { X, Aguilera D, Zhao J(23) }\end{array}$ & $\begin{array}{l}\text { Relato da crença religiosa, analisadas de } \\
\text { forma dicotômica. }\end{array}$ & $\begin{array}{l}\text { SBQ-R - Questionário de comportamento suicida - } \\
\text { revisado }^{(32)}\end{array}$ \\
\hline $\begin{array}{l}\text { Band M, Dein S, Loewenthal } \\
\mathrm{KM}^{(24)}\end{array}$ & Para você, o que significa ser religioso? & $\begin{array}{l}\text { - Como suas crenças religiosas afetam sua vida? } \\
\text { - Seus valores religiosos o ajudam a lidar com eventos } \\
\text { - Você acha que existem circunstâncias em que o suicídio } \\
\text { pode ser aceitável? } \\
\text { - Você acha que suas crenças religiosas o ajudam a lidar } \\
\text { com questões de vida e morte? } \\
\text { - Alguma vez as coisas ficaram tão ruins que você já } \\
\text { pensou em se prejudicar de alguma maneira? } \\
\text { - Você acha que a religião influencia o modo como as } \\
\text { pessoas se sentem sobre o suicídio? } \\
\text { - Quando você fica sabendo que alguém cometeu suicídio, } \\
\text { as questões de religiosidade afetam como você vê suas } \\
\text { ações? } \\
\text { - Se você já esteve em um estado de desespero, você } \\
\text { acha que suas crenças religiosas ajudaram a lidar com } \\
\text { essa situação? }\end{array}$ \\
\hline Rasic D, Kisely S, Langille DB(25) & $\begin{array}{l}\text { Importância da religião e a participação nos } \\
\text { serviços religiosos, analisadas de forma } \\
\text { dicotômica. }\end{array}$ & $\begin{array}{l}\text { Relato de experiência anterior de comportamento suicida no } \\
\text { último ano }\end{array}$ \\
\hline
\end{tabular}

Figura 4 - Principais instrumentos e questionamentos utilizados para avaliar a religião/espiritualidade e o comportamento suicida e os respectivos autores que validaram ou construíram as ferramentas 
Com relação à avaliação do comportamento suicida nos estudos quantitativos, constatou-se também a aplicação de diferentes instrumentos ${ }^{(20-23,25)}$.

Quanto aos estudos de abordagem qualitativa, a pesquisa conduzida com universitárias utilizou perguntas direcionadas às situações estressoras como suicídio e a relação da espiritualidade como força e apoio. É prudente mencionar que esse artigo realizou uma triagem utilizando três instrumentos, como a Escala de Ideação Suicida (SSI), o Questionário de comportamento suicida - revisado (SBQ-R), e o Inventário de Resiliência ao Suicídio (SRI-25). A pesquisa foi conduzida apenas com mulheres universitárias que apresentavam alteração para o comportamento suicida(19). O outro estudo avaliou através de diversas questões a relação entre a religiosidade e o suicídio(24).

Os principais resultados dos estudos referentes à relação entre R/E e o comportamento suicida encontra-se na Figura 5.

No que diz respeito aos resultados dos artigos, identificou-se que existem quatro $(57,1 \%)$ estudos que apoiam a associação entre a R/E e a prevenção do comportamento suicida, sendo que em dois estudos essa relação positiva foi encontrada apenas para os adolescentes cristãos ${ }^{(21)}$ e na outra, apenas no sexo feminino(25). Dois estudos não encontraram uma relação entre R/E e o enfrentamento para o comportamento suicida. Contudo, o compromisso religioso contribuiu indiretamente para que os jovens fossem mais compreensivos em relação ao comportamento suicida de outras pessoas e considerassem que o mesmo pode ser prevenido(22), e que a fé também pode contribuir para os jovens atravessarem o período de situações difíceis, mas não é suficiente para ativar em situações agudas de estresse e trauma ${ }^{(24)}$.

Um fato importante a ser elucidado é que um dos estudos referenciou que a religião pode não ser um protetor universal contra ideação suicida em diferentes religiões, e que o desejo de viver foi um fator protetor contra o comportamento suicida, independente da religião(21).

É relevante mencionar que em um dos artigos houve efeito inverso, em que ser religioso aumentou o risco do suicídio. Os autores explicam que os jovens, ao acreditarem em uma vida após a morte, veem no suicídio uma forma rápida de acelerar essa conquista(23).

Quanto à explicação dos benefícios da $R / E$, apenas dois artigos retratam que a conexão com a divindade proporciona uma sensação de conforto, alívio e vigor para continuar vivendo(19) e que quanto mais religioso o jovem for mais ele é seduzido pela vida(20).

\section{Relação entre religião/espiritualidade e o comportamento suicida}

Conclui-se que os aspectos protetores da espiritualidade na promoção da adaptação e do bem-estar na vida dos estudantes em risco de suicídio através de suas percepções de Deus, oferecendo um significado importante da espiritualidade no aumento da resiliência ao suicídio. Conexão com Deus como uma "presença" e 'força' no meio das adversidades proporciona aos indivíduos uma sensação de conforto, alívio e vigor para continuar com a vida. Essas consequências significativas representam os aspectos positivos dos comportamentos de enfrentamento que podem estar bem associados à resiliência ou adaptação positiva( ${ }^{(19)}$.

Existe uma correlação negativa $(-0,227)$ significativa no nível de 0,005 entre atração pela vida e o Teste de Orientação Religiosa, isso indica que quanto mais religiosa o jovem é, mais atraído pela vida ele será. Conclui-se que a religião atua como fator de proteção contra o suicídio(20).

Uma correlação negativa significativa $(r=-0,33)$ foi encontrada entre nível de religiosidade e ideação suicida, mas apenas entre os adolescentes cristãos. A devoção religiosa pode não ser uma proteção universal contra a ideação suicida, em diferentes religiões ${ }^{(21)}$.

A aceitação do suicídio variou significativamente $(0,01)$ entre os grupos religiosos, sendo que os participantes budistas $($ Média=24,8) têm maior aceitação em comparação aos muçulmanos (Média=19,9). A participação dos universitários em diferentes grupos religiosos não diferenciou quanto a probabilidade de acreditar que o suicídio era evitável e também em relação ao risco de suicida. Contudo, o resultado da análise de regressão logística evidenciou que a probabilidade de tentativa suicídio entre os universitários pode ser prevista por sua etnia e afiliação religiosa, os chineses e budistas têm 2,7 vezes mais chances de tentativa de suicida do que os malaios e muçulmanos. A religião na vida dos estudantes ajuda a moldar as normas sobre o suicídio nos jovens. A influência das atitudes em relação ao suicídio e a religião, deve ser considerada no desenvolvimento de programas de prevenção do suicídio(22)

Ter crença religiosa foi significativamente relacionada ao risco suicídio pela matriz de coeficiente de correlação de Pearson. Portanto, ser religioso estava associado a aumento do risco de suicídio, uma vez que ao acreditarem na vida após a morte, o suicídio seria um meio de acelerar essa conquista ${ }^{(23)}$.

Conclui-se que existe ambiguidade na relação entre religiosidade, enfrentamento e suicídio. Refere que ser religioso não garante automaticamente que seja um elemento de enfrentamento e que a fé e a crença não são ativadas em situações agudas de estresse e trauma. Todavia, a fé é uma fonte de conforto e contribuiu para que alguns participantes superassem situações difíceis ${ }^{(24)}$.

Entre as mulheres, a menor importância pessoal da religião esteve associada à 1,8 vezes mais chances à ideação suicida, sendo estatisticamente significante $(p<0,001)$ e 1,5 vezes mais chance para a tentativa de suicídio, não havendo diferença significativa. Sendo que pouca participação religiosa apresentou 1,8 vezes mais chance para ideação suicida, estatisticamente significante $(p<0,05)$ e 1,4 vezes mais chance para tentativa de suicídio, não foi estaticamente significante ${ }^{(25)}$.

Figura 5 - Principais resultados dos estudos analisados referentes a relação entre religião/espiritualidade e o comportamento suicida 


\section{Discussão}

Constatou-se uma lacuna de estudos no contexto nacional referente à relação entre $R / E$ e o comportamento suicida em adolescentes e jovens, o que já vem sendo retratado na literatura(33). Neste sentido, é necessário que pesquisas sejam conduzidas com esse grupo, uma vez que as taxas de suicídio nessa população são expressivas( ${ }^{(34)}$, além do que a R/E faz parte da cultura, da socialização e da rotina diária de muitos brasileiros( ${ }^{(35)}$, sendo o Brasil um país eminentemente religioso. Um maior investimento nessas pesquisas pode contribuir tanto para compreendermos melhor as estratégias de prevenção ao suicídio empregadas por essa população, bem como as possíveis particularidades em relação ao contexto brasileiro. Nesse sentido, a R/E pode se revelar um fator protetivo mais ou menos expressivo em função desse contexto, o que ainda deve ser melhor investigado e suportado por mais evidências científicas.

A principal abordagem realizada pelos pesquisadores nesse campo foi a quantitativa, e em nenhum dos estudos realizou o cálculo estatístico para definição da amostra. A realização do cálculo amostral contribui para a validade interna do estudo, sendo de fundamental relevância que seja descrita nos artigos(36).

Outro fato importante a ser destacado é que grande parte dos estudos foi conduzida em universidades e com os universitários. Tal situação pode estar relacionada com o acesso facilitado aos estudantes, bem como ao sofrimento vivenciado nesse contexto social e por esse grupo, como a violência, a síndrome do pânico, o estresse, a depressão e o suicídio, além do que o mundo muitas vezes se tornou desinteressante e sem sentido para os jovens(37). A literatura referencia que os estudantes universitários são um grupo que merece atenção em relação ao comportamento suicida( ${ }^{(38)}$. Além disso, o contexto institucional vem cada vez mais sendo alvo de reflexões em termos da saúde mental, sendo necessário que as instituições de ensino superior estejam engajadas não apenas em programas de atendimento a esses estudantes, mas que também possam desenvolver ações de acolhimento a esse público, o que perpassa, as reflexões sobre a prevenção ao comportamento suicida nessa etapa da formação.

Pesquisa realizada com 637 universitários, com idade variando entre 18 a 32 anos, da Universidade Federal de Mato Grosso, detectou que 9,9\% apresentavam ideias suicidas nos últimos 30 dias $^{(33)}$. Investigação também conduzida com 220 universitários da Irlanda observou que $59 \%$ dos participantes apresentaram sintomas depressivos e $28,5 \%$ ideação suicida(39). Esses dados convergem com a pesquisa realizada com 523 estudantes de graduação da Etiópia, que encontrou que a estimativa geral de comportamento suicida foi de $28,9 \%$, enquanto que a prevalência ao longo da vida de ideação foi de $58,3 \%$, plano suicida de $37,3 \%$, e a tentativa de suicídio de $4,4 \%{ }^{(38)}$.

Há que se considerar que dois artigos incluídos na amostra da presente pesquisa tiveram como cenário a escola, e a população, adolescentes do ensino médio. Esse grupo também apresenta taxas significativas de comportamento suicida, conforme evidenciado em estudo realizado em Gana, no qual os adolescentes apresentaram maiores taxas de estresse, depressão e ideação suicida do que os estudantes universitários(40). Pesquisa com adolescentes do ensino médio do Irã concluiu que $4,1 \%$ apresentavam pensamentos suicidas no último 12 meses $^{(41)}$. No contexto brasileiro, também evidenciou-se que, $12,1 \%$ dos adolescentes do ensino médio tiveram ideações suicidas e 5,5\% tentaram suicídio nos últimos 12 meses, sendo que a maior prevalência de ideação foi na faixa etária entre 14 a 16 anos(34). $^{(34)}$

É imperativo que pesquisas sejam conduzidas nos espaços das escolas e das universidades e que os profissionais de saúde estejam aptos a implementar estratégias que possam acolher os adolescentes e jovens em toda a sua dimensão existencial, contribuindo para melhoria do seu bem-estar(37,40). Embora a literatura recuperada não explore suficientemente o papel da gestão educacional, é mister que os dirigentes dessas instituições de ensino, tanto do ensino médio quanto do superior, estejam engajados em ações de promoção de saúde mental que possam considerar, de modo enfático, a prevenção ao comportamento suicida. Essas estratégias também podem se dar de modo articulado a outras ações de acolhimento fundamentais para esse público.

Quanto à idade dos participantes, considerando os critérios de inclusão estabelecido no presente estudo, constatou-se que a idade variou de 15 a 24 anos. Fato importante de ser elucidado que mundialmente a segunda principal causa de morte entre os jovens de 15 a 29 anos se refere ao suicídio, e que ainda é uma área da saúde negligenciada(41). Com relação a essa variável, observa-se que pesquisas foram excluídas do presente estudo em decorrência da idade ser superior a 25 anos $^{(11,42,43)}$. Em algumas investigações os autores apenas mencionaram que os participantes tinham mais de $18 \operatorname{anos}^{(44,45)}$, ou que eram adolescentes e jovens adultos $^{(46)}$, e outros retratavam apenas a média de idade dos jovens ${ }^{(38,47)}$. Recomenda-se que os estudos especifiquem claramente a variação da idade dos participantes para permitir melhor compreensão dos resultados.

É oportuno apontar que estudos com um grupo etário muito amplo podem alterar os resultados das 
pesquisas em relação à vivência da $\mathrm{R} / \mathrm{E}^{(42-43)}$, assim como do comportamento suicida. Sabe-se que os adolescentes e jovens estão expostos a outras vulnerabilidades, como o consumo de álcool, tabaco e outras substâncias, depressão, violência entre os pares e demais aspectos que seriam catalisadores para elevar a taxa de suicídio entre os demais grupos.

O que coaduna com investigação conduzida com participantes entre 18 a 36 anos, que o risco de suicídio é maior nos mais jovens, os quais eram menos comprometidos com a religião, apresentaram maiores taxas de sintomas de depressão, de ansiedade, consumo de álcool, percebiam menos apoio social da família e de amigos e não procuravam ajuda, sendo que essas variáveis eram significantes ${ }^{(11)}$.

Outra questão relevante refere-se ao fato de que a maioria dos artigos estudou apenas as questões do sexo/ gênero e a relação com o suicídio, o que corrobora com a investigação que descreve a negligência das pesquisas sobre a interface entre $R / E$ e a saúde mental da comunidade LGBTQI+, grupo com risco aumentado para o comportamento suicida(33,48). Os autores consideram que é necessário mais pesquisas com população LGBTQI+(49) e que as mesmas sejam conduzidas com objetivo de definir intervenções que contribuam para a prevenção do suicídio(48). Deve-se destacar, ainda, que a R/E pode ser um fator de proteção importante, notadamente quando se mostra acolhedora com minorias sexuais e de gênero(50), permitindo que jovens LGBTQI+ que estejam conectados com a dimensão da R/E também possam adotar comportamentos mais saudáveis, o que envolve também a prevenção ao comportamento suicida.

Relacionado às particularidades da dimensão da R/E com o construto comportamento suicida, constatou-se que a maioria dos estudos tinha como objetivo investigar essa relação. Para ambos os construtos, os pesquisadores utilizaram diferentes instrumentos para mensurar as variáveis. Apenas um estudo qualitativo investigou a dimensão espiritualidade, e os demais avaliaram a crença, a importância da religião, rituais religiosos e a participação no serviço religioso.

Quanto à investigação do comportamento suicida, um estudo quantitativo(23) aplicou o SBQ-R - Suicidal Behaviors Questionaire- Revised e em outro este mesmo instrumento foi utilizado para identificar os participantes com comportamento suicida e posteriormente conduzir a abordagem qualitativo(19). Apresenta-se como uma ferramenta fidedigna para a população adolescente e adulta, de autorrelato, tipo likert, em que se avalia o risco de suicídio através de quatro questões, com pontuação que varia de três a 18 pontos, um escore igual ou superior a sete o indivíduo apresenta alto risco para comportamento suicida ${ }^{(32,11)}$.
Os resultados dos artigos evidenciaram discrepâncias do nexo entre os construtos da R/E com o comportamento suicida. Pode-se inferir que a divergência dos resultados pode estar relacionada aos diferentes instrumentos empregados(47) e as metodologias, além das questões socioculturais, das diferentes religiões e como os jovens vivenciam a dimensão da religião e da espiritualidade em suas vidas.

Apesar da divergência dos resultados, os artigos retrataram os benefícios da religião e da $R / E$, mesmo que indiretamente, na vida dos jovens em relação ao suicídio. E que a gênese para esse benefício está no conforto, no alívio de situações estressantes e na satisfação pela vida. A R/E tem um efeito positivo na saúde mental do ser humano, haja vista que aumenta as emoções positivas e reduz o estresse que poderia gerar sentimentos negativos como, por exemplo, a ideação suicida. A R/E também contribui para definir as doutrinas da vida em sociedade e o comportamento pró-social: ao cumpri-las o indivíduo reduz a probabilidade de vivenciar situações geradores de estresse. A R/E também pode ser promotora de virtudes humanas como honestidade, perdão, gratidão e paciência, que aumentam as emoções positivas $^{(13)}$, funcionam como fatores protetivos em termos de saúde mental.

Outras pesquisas retratam que a R/E contribui para o bem-estar(33,35), uma vez que esta dimensão propicia uma rede de apoio social o que minimiza a solidão e a sensação de isolamento, e, simultaneamente, muitos ensinamentos religiosos nutrem a ideia de que o suicídio não é uma alternativa viável para lidar com sofrimentos e problemas ${ }^{(35)}$. Os efeitos dessa associação podem ser encontrados em outros estudos, como o que foi conduzido com universitários que também autopreencheram o SBQ-R, concluindo que somente as jovens mulheres que tinham menos envolvimento com a prática religiosa apresentavam maiores chances para o risco de suicídio(38). Pesquisa conduzida no contexto brasileiro detectou que os universitários que não tinham uma prática religiosa apresentavam maior risco de comportamento suicida(33).

Esses dados convergem com pesquisa desenvolvida com universitários americanos em que observou que a religiosidade intrínseca (crenças e práticas religiosas) foi fator protetor para prevenção da ideação suicida apenas nas mulheres ${ }^{(47)}$. Evidencia-se também em uma investigação com indivíduos adultos do Irã que tentaram suicídio em comparação com aqueles que não, que a religião intrínseca, como as crenças religiosas estão relacionadas a prevenção do comportamento suicida, contudo, a prática religiosa não apresentou correlação com essa variável(51).

Os resultados de um estudo com adolescentes e jovens adultos em Trinidad e Tobago detectou 
associações protetoras do pensamento suicida apenas entre os que se autodeclararam católicos e adventista do sétimo dia. A maior religiosidade autoavaliada pelos participantes esteve relacionada à menor probabilidade de pensar suicídio e de planejar suicídio. Assim, como o comparecimento a serviços religiosos estava associado à diminuição do risco de pensamentos e de tentativa de suicídio. Observou-se também uma associação protetora entre maior frequência de oração e menor chances de pensamento e planos suicida, o que contribui para minimizar o estresse e o sofrimento(46).

Há relatos em um dos artigos que compôs a amostra do estudo, que revelam que, mesmo que tenha fé, nem sempre o jovem a emprega no enfrentamento de adversidades, apesar de alguns acreditarem que é fundamental para sobreviver a esses momentos. Tais resultados coadunam com investigação qualitativa com jovens e adultos que haviam tentado o suicídio, os mesmos consideram a existência de Deus, e que Ele é o responsável por presentear com a vida e de recolhê-la. Também acreditam na relevância da religião para saúde mental e o bem-estar, contudo, eles fracassaram em usá-la durante o sofrimento e que todo esse processo gerou sentimento de culpa e de reprovação pelo ato(43).

\section{Conclusão}

A partir dos estudos recuperados na presente revisão, evidencia-se que a $\mathrm{R} / \mathrm{E}$ é um elemento relevante na vida dos adolescentes e dos jovens. Entretanto, é necessário que mais pesquisas sejam conduzidas e direcionadas a compreender a vivência da dimensão desses construtos na vida dos mesmos e de seus efeitos, sobretudo em relação ao comportamento suicida. Embora as evidências recuperadas neste estudo sejam limitadas em termos de seu poder de efeito para comparações mais robustas, apenas sete estudos foram recuperados, há que se destacar que, em sua maioria, apontam para efeitos protetivos da R/E em relação à prevenção do comportamento suicida. Assim, a R/E pode ser evocada não apenas como um recurso em saúde mental, o que se alinha, inclusive, às recomendações da Organização Mundial da Saúde, a partir do seu conceito multidimensional de saúde, atualizado em 1998, mas a um elemento que pode ser mais discutido com os adolescentes e jovens.

Em uma sociedade cada vez mais hiperconectada, automatizada e com pouco espaço para incursões no universo do próprio indivíduo, a R/E pode ser um convite para que esses jovens estejam em contato com as próprias sensações, com os próprios recursos e com reflexões que estejam para além da materialidade, o que pode evocar respostas mais seguras e alinhadas a uma real promoção de saúde pela possibilidade de retorno ao que, de fato, os constitui. Uma necessidade deflagrada a partir da presente revisão é a de compreender como esse público compreende e vivencia a R/E. No cenário brasileiro, eminentemente religioso e com diversas manifestações religiosas e espirituais, abre-se a possibilidade de que os estudos no campo da saúde mental devam estar em constante diálogo com aqueles produzidos pelas ciências humanas, em busca de apreensão do próprio fenômeno religioso nessa faixa etária e suas especificidades a partir dos diferentes marcadores sociais como origem, renda, escolaridade e acesso à saúde. Essas relações podem se mostrar relevantes, em estudos vindouros, para uma apreensão mais profunda acerca do papel protetivo da R/E sobre o comportamento suicida. Essas reflexões podem fazer parte de programas e ações de prevenção ao suicídio não como forma de doutrinação ou de exploração de uma atuação profissional considerada religiosa, pelo contrário: como possibilidade de levar o indivíduo a uma conexão interna que é promotora de autoconhecimento e, consequentemente, de autocuidado, tendo como referência uma $\mathrm{R} / \mathrm{E}$ inteligível e prenhe de sentido para esse público.

\section{Referências}

1. World Health Organization. Preventing suicide a global imperative. Geneva: WHO; 2014. [cited Mar 15 2020]. Available from: http://www.who.int/ mental_health/suicide-prevention/world_report_2014/ en/

2. Goodfellow B, Kõlves K, de Leo D. Contemporary Definitions of Suicidal Behavior: A Systematic Literature Review. Suicide Life Threat Behav. 2018;49(2):488-504. https://doi.org/10.1111/sltb.12457

3. Penso MA, Sena DPA de. Young people's hopelessness and suicide as a solution. Soc Estado. 2020;35(1):61-81. doi: https://doi.org/10.1590/ s0102-6992-202035010004

4. Orellana JDY, Ribeiro MRC, Barbieri MA, Saraiva MC, Cardoso VC, Bettiol $\mathrm{H}$, et al. Mental disorders in adolescents, youth, and adults in the RPS Birth Cohort Consortium (Ribeirão Preto, Pelotas and São Luís), Brazil. Cad Saúde Pública. 2020;36 (2): e00154319. doi: https://doi.org/10.1590/0102-311x00154319

5. Oliver A, Galiana L, Sancho P, Tomás J M. Spirituality, hope and dependence as predictors of satisfaction with life and perception of health: the moderating Effect of being very old. Aquichan. 2015;15(2):228-38. doi: https://dx.doi.org/10.5294/aqui.2015.15.2.7

6. Oliveira MR, Junges JR. Mental health and spirituality/ religiosity: psychologists' understandings. Estud Psicol. 2012;17(3):469-76. doi: doi.org/10.1590/ S1413-294X2012000300016 
7. Scorsolini-Comin F. The religiosity/spirituality in health [editorial]. Rev Ciênc Saúde. 2018;8(2):1-2. doi: https://doi.org/10.21876/rcsfmit.v8i2.752

8. Cunha VFF, Scorsolini-Comin F. The Religiousness/ Spirituality Dimension in Clinical Practice: An Integrative Review of the Scientific Literature. Psicol Teor Pesq. 2019;35:e35419. doi: https://doi. org/10.1590/0102.3772e35419

9. Inoue TM, Vecina MVA. Spirituality and/or religiosity and health: a literature review. J Health Sci Inst. 2017;35(2):127-30. [cited Mar 10 2020]. Available from: https://www.unip.br/presencial/comunicacao/ publicacoes/ics/edicoes/2017_02.aspx

10. Précoma $D B$, Oliveira GMM, Simão $A F$, Dutra $O P$, Coelho OR, Izar COM, et al. Atualização da Diretriz de Prevenção Cardiovascular da Sociedade Brasileira de Cardiologia - 2019. Arq Bras Cardiol. 2019;113(4):787891. doi: 10.5935/abc.20190204

11. Hamdan S, Peterseil-Yaul T. Exploring the psychiatric and social risk factors contributing to suicidal behaviors in religious young adults. Psychiatry Res. (Internet). 2019;20:112449. [cited Mar 10 2020]. Available from: https://www.ncbi.nlm.nih.gov/pubmed/31229309

12. Cunha VF, Scorsolini-Comin F. Best professional practices when approaching religiosity/spirituality in psychotherapy in Brazil. Couns Psychother Res. 2019;19:523-32. doi: https://doi.org/10.1002/ capr. 12241

13. Koenig HG. Religion, spirituality, and health: the research and clinical implications. ISRN Psychiatry. 2012;2012:278730. doi: https://doi. org/10.5402/2012/278730

14. Mendes KDS, Silveira RCCP, Galvão CM. Integrative literature review: a research method to incorporate evidence in health care and nursing. Texto Contexto Enferm. 2008;17(4):758-64. [cited Mar 17 2020]. Available from: https://www.scielo.br/scielo. php?pid=s0104-07072008000400018\&script=sci_arttext 15. Santos CMC, Pimenta CAM, Nobre MRC. The PICO strategy for the research question construction and evidence search. Rev. Latino-Am. Enfermagem. 2007;15(3):508-11. doi: https://doi.\org/10.1590/ S0104-11692007000300023

16. World Health Organization. Young people's health a challenge for society: report of a WHO Study Group on Young People and "Health for All by the Year 2000". Technical Report Series 731. Geneva: WHO; 1986. https://apps.who.int/iris/handle/10665/41720

17. Ministério da Saúde. Secretaria de Atenção à Saúde (BR). Área de Saúde do Adolescente e do Jovem. Marco legal: saúde, um direito de adolescentes. Brasília: Ministério da Saúde; 2007. 60 p.[Acesso 28 jul 28 2020 ].Disponível em: http://www.adolescencia.org.br/ upl/ckfinder/files/pdf/marco_legal.pdf
18. Felipe AOB, Carvalho AMP, Andrade CUB. Spirituality and religion as protectors for adolescente drug use. SMAD, Rev. Eletrônica Saúde Mental Álcool Drog. 2015; 11(1):49-58. doi: http://dx.doi.org/10.11606/ issn.1806-6976.v11i1p49-58

19. Francis W, Bance LO. Protective Role of Spirituality from the Perspective of Indian College Students with Suicidal Ideation: "I am here Because God Exists". J Relig Health. 2017; 56: 962-70. doi: https://doi. org/10.1007/s10943-016-0296-6

20. Kazi T, Naidoo S. Does Religiosity Mediate Suicidal Tendencies? A South African Study of Muslim Tertiary Students. J Relig Health. 2016;55:1010-23. doi: 10.1007/s10943-015-0167-6

21. Muallem HK, Israelashvilli M. Religiosity as a buffer against suicidal ideation: a comparison between Christian and Muslim-Arab adolescentes. Mental Health, Religion Culture. 2015;18(10): 838-49. doi: http://dx.doi.org/1 $0.1080 / 13674676.2015 .1107891$

22. Foo XY, Alwi MNM, Ismail SIF, Ibrahim N, Osman ZJ. Religious Commitment, Attitudes Toward Suicide, and Suicidal Behaviors Among College Students of Different Ethnic and Religious Groups in Malaysia. J Relig Health. 2014;53:731-46. doi:10.1007/s10943-012-9667-9

23. Zhao J, Yang $X$, Xiao R, Zhang $X$, Aguilera $D$, Zhao J. Belief system, meaningfulness, and psychopathology associated with suicidality among Chinese college students: a cross-sectional survey. BMC Public Health. 2012;12:668. doi: 10.1186/1471-2458-12-668

24. Band M, Dein S, Loewenthal KM. Religiosity, coping, and suicidality within the religious Zionist community of Israela thematic qualitative analysis. Mental Health, Religion Culture. 2011;14(10):1031-47. doi: https://doi. org/10.1080/13674676.2010.548059

25. Rasic D, Kisely S, Langille DB. Protective associations of importance of religion and frequency of service attendance with depression risk, suicidal behaviours and substance use in adolescents in Nova Scotia, Canada. J Affective Disorders. 2011;132(3):389-95. doi: https:// doi.org/10.1016/j.jad.2011.03.007

26. Idehen EE. The development and evaluation of a religiosity scale. IFE Psychologia. 2001;9:58-69. doi: 10.4314/ifep.v9i2.23617

27. Orbach I, Milstein I, Har-Even D, Apter A, Tiano S, Elizur A. A multi-attitude suicide tendency scale for adolescents. J Consult Clin Psychol. 1991;3(3):398-404. 28. Katz $A$. The link between religious identity, Israeli identity, and value identification with military service among Jews and Druze in Israel (Unpublished master's thesis). Tel Aviv: University Education; 2000.

29. Reynolds WM. Psychometric characteristics of the adult suicidal ideation questionnaire in college students. Personal Assess. 1991;56(2):289-307. doi: https://doi. org/10.1207/s15327752jpa5602_9 
30. Worthington E, Wade N, Hight T, Ripley J, McCullough M, Berry J, et al. The religious commitment inventory-10: Development, refinement, and validation of a brief scale for research and counselling. J Counsel Psychol. 2003 Jan 1;50(1):84-96. doi: https://doi. org/10.1037/0022-0167.50.1.84

31. Salander-Renberg E, Jacobsson L. Development of a questionnaire on attitudes towards suicide (ATTS) and its application in a Swedish population. Suicide Life-Treat Behav. 2003;33:52-64. doi: 10.1521/suli.33.1.52.22784 32. Osman A, Bagge CL, Guitierrez PM, Konick LG, Kooper BA, Barrios FX. The suicidal behaviour questionnaire-revised (SBQ-R): validation with clinical non clinical samples. Assessment. 2001;5:443-54. doi: 10.1177/107319110100800409

33. Santos HGB, Marcon SR, Espinosa MM, Baptista MN, Paulo PMC. Factors associated with suicidal ideation among university students. Rev. Latino-Am. Enfermagem [Internet]. 2017;25:e2878. [cited Mar 19 2020]. Available in: http://www.scielo.br/scielo.php?script=sci_arttext\&p id=S0104-11692017000100332.

34. Alves CAS Junior, Nunes HEG, Gonçalves ECA, Silva DAS. Suicidal behaviour in adolescents: characteristics and prevalence. J Hum. Growth Dev. 2016;26(1):88-94. [cited Mar 27 2020]. Available in: http://pepsic.bvsalud.org/scielo.php?script=sci_ arttext\&pid=S0104-12822016000100013\&lng=pt\&nrm= iso

35. Nantes AC, Grubits S. A religiosidade/espiritualidade como um possível fator de ajuda à prevenção da prática suicida. Rev Contemplação. 2017;16:73-84. [Acesso 27 mar 2020]. Disponível em: http://fajopa.com/ contemplacao/index.php/contemplacao/article/view/155 36. Brito CJ, Grigoletto MES, Toledo Nóbrega O, Córdova C. Sample size and the myth of magical numbers: point of view. Rev Andaluza Medicina Deporte. 2016;9(1):29-31. [cited Mar 22 2020]. Available in: https://www.sciencedirect.com/science/article/pii/ S1888754615001318

37. Dutra E. University student's suicide: existential emptiness in contemporary times. Estud Pesqui Psicol. [Internet]. 2012;12(3):924-37. [cited Mar 20 2020]. Available in: http://pepsic.bvsalud.org/scielo.php?script=sci_ arttext\&pid=S1808-42812012000300013\&lng=pt\&nrm= iso

38. Abdu Z, Hajure M, Desalegn D. Suicidal Behavior and Associated Factors Among Students in Mettu University, South West Ethiopia, 2019: An Institutional Based CrossSectional Study. Psychol Res Behav Manage. 2020; 13: 233-43. doi: 10.2147/PRBM.S240827

39. Horgan A, Kelly P, Goodwin J, Behan L. Depressive Symptoms and Suicidal Ideation among Irish Undergraduate College Students. Issues Ment Health
Nurs. 2018; 39(7):575-84.[cited Mar 20 2020]. Available from: https://www.ncbi.nlm.nih.gov/pubmed/29465285 40. Ahorsu DK, Adjaottor ES, Yeboah FA, Opoku Y. Mental health challenges in academia: comparison between students of the various educational levels in Ghana. J Ment Health. 2020;13:1-8. [cited Mar 21 2020]. Available from: https://doi.org/10.1080/096382 37.2020 .1739253

41. Ziaei R, Viitasara E, Soares J, Sadeghi-Bazarghani $\mathrm{H}$, Dastgiri S, Zeinalzadeh AH, Bahadori F, Mohammadi R. Suicidal ideation and its correlates among high school students in Iran: a cross-sectional study. BMC Psychiatry. 2017; 17: 147. [cited Mar 21 2020]. Available from: https://www.ncbi.nlm.nih.gov/pmc/ articles/PMC5397734/

42. Lytle MC, Blosnich JR, Susan M, De Luca SM, Brownson C. Association of Religiosity With Sexual Minority Suicide Ideation and Attempt. Am J Prev Med. 2018; 54(5):64465. doi: 10.1016/j.amepre.2018.01.019

43. Akotia CS, Knizek BL, Kinyanda E, Hjelmelan H. "I have sinned": understanding the role of religion in the experiences of suicide attempters in Ghana. Mental Health, Religion Culture. 2014; 17(5): 437-48. http:// dx.doi.org/10.1080/13674676.2013.829426

44. Kleiman EM, Liu RT. Prospective prediction of suicide in a nationally representative sample: religious service attendance as a protective factor. $\mathrm{Br} \mathrm{J}$ Psychiatry. 2014;204:262-6. doi: 10.1192/bjp.bp.113.128900

45. Wang Z, Koenig Harold G, Ma W, Liu L. Religious involvement, suicidal ideation and behavior in mainland China. Int J Psychiatry Med. 2015;48(4):299-316. doi: 10.2190/PM.48.4.e

46. Toussaint L, Wilson CM, Wilson LC, Williams DR. Williams. Religiousness and suicide in a nationally representative sample of Trinidad and Tobago adolescents and young adults. Soc Psychiatry Psychiatr Epidemiol. 2015; 50(9):1441-50. doi:10.1007/s00127-015-1045-y 47. Lester D, Walker RL. Religiosity Is a Protective Factor for Suicidal Ideation in European American Students but Not in African American Students. OMEGA- J Death Dying. 2017;74(3):295-303. doi: 10.1177/0030222815598452 48. Meyer IH, Teylan M; Schwartz S. The Role of HelpSeeking in Preventing Suicide Attempts among Lesbians, Gay Men, and Bisexuals. Suicide Life Threat Behav. 2015;45(1):25-36. doi:10.1111/sltb.12104.

49. Baere F, Zanello V. The gender in suicidal behavior: An epidemiological reading of data from the Federal District. Estud Psicol. 2018;23(2):168-78. http://dx.doi. org/10.22491/1678-4669.20180017

50. Ribeiro LM, Scorsolini-Comin F. Relations between religiousness and homosexuality in religious young adults. Psicol Soc. 2017;29:e162267. doi: https://doi. org/10.1590/1807-0310/2017v29162267 
51. Baneshi MR, Haghdoost AA, Zolala F, Nakhaee N, Jalali M, Tabrizi R, et al. Can Religious Beliefs be a Protective Factor for Suicidal Behavior? A Decision Tree Analysis in a Mid-Sized City in Iran, 2013. J Relig Health. 2017;56:428-36. doi: 10.1007/s10943-016-0215-x

\section{Contribuição dos autores}

Concepção e planejamento do estudo: Maria Betânia Tinti de Andrade, Adriana Olimpia Barbosa Felipe e Fabio Scorsolini-Comin. Obtenção dos dados: Maria Betânia Tinti de Andrade e Adriana Olimpia Barbosa Felipe. Análise e interpretação dos dados: Maria Betânia Tinti de Andrade, Adriana Olimpia Barbosa Felipe e Kelly Graziani Giacchero Vedana. Redação do manuscrito: Maria Betânia Tinti de Andrade, Adriana Olimpia Barbosa Felipe, Kelly Graziani Giacchero Vedana e Fabio Scorsolini-Comin. Revisão crítica do manuscrito: Maria Betânia Tinti de Andrade, Adriana Olimpia Barbosa Felipe, Kelly Graziani Giacchero Vedana e Fabio Scorsolini-Comin.

Todos os autores aprovaram a versão final do texto.

Conflito de interesse: os autores declararam que não há conflito de interesse. 